\title{
COUNTABLE PARACOMPACTNESS IN PRODUCT SPACES
}

\section{PHILLIP ZENOR}

\begin{abstract}
The main purpose of this paper is to show that $X^{\omega}$ is normal if and only if (1) $X^{n}$ is normal for each $n$, and (2) $X^{\omega}$ is countably paracompact. Furthermore, $X^{\omega}$ is perfectly normal if and only if $X^{\omega}$ is hereditarily countably paracompact. Also, the compact Hausdorff space $X$ is metrizable if and only if $X^{3}$ is hereditarily countably paracompact.
\end{abstract}

This note was prompted by [4], where Michael offers an example of a space $X$ such that for each $n, X^{n}$ is paracompact, but $X^{\omega}$ is not normal. Following the current notation, $X^{n}$ denotes the topological product of $n$ copies of $X$ and $X^{\omega}$ denotes the product of countably many copies of $X$. For each integer $n, \odot_{n}$ denotes the projection of $X^{\omega}$ onto $X^{n}$. Throughout this paper, our spaces are assumed to be Hausdorff. The following lemma is due to Ishikawa [2]:

Lemma A. The space $X$ is countably paracompact if and only if for each monotonic decreasing sequence $\left\{H_{n}\right\}$ of closed sets with no common part there is a sequence $\left\{D_{n}\right\}$ of open sets such that $H_{n} \subset D_{n}$ for each $n$ and such that $\bigcap_{n=1}^{\infty} \mathrm{cl} D_{n}=\varnothing$.

The next lemma was established in [5]:

LEMma B. The space $X$ is normal if for each pair $(H, K)$ of mutually exclusive closed subsets of $X$ there is a sequence $\left\{D_{n}\right\}$ of domains such that $H \subset \bigcap_{n=1}^{\infty} D_{n} \subset \bigcap_{n=1}^{\infty} \mathrm{cl} D_{n} \subset X-K$.

THEOREM A. $X^{\omega}$ is normal if and only if (1) $X^{n}$ is normal for each $n$ and (2) $X^{\omega}$ is countably paracompact.

Proof. First, suppose that each $X^{n}$ is normal and $X^{\omega}$ is countably paracompact. Let $H$ and $K$ denote mutually exclusive closed subsets of $X^{\omega}$. The object is to obtain a sequence of domains, $\left\{D_{n}\right\}$, each element of which contains $H$ such that $\left\{D_{n}\right\}$ satisfies our Lemma B. Note that if for some integer $n, \operatorname{cl} \odot_{n}(H) \cap \mathrm{cl} \rho_{n}(K)=\varnothing$, then there are mutually exclusive open sets $U$ and $V$ in $X^{n}$ such that $U$ contains cl $\rho_{n}(H)$ and $V$ contains $\mathrm{cl} \odot_{n}(K)$. For each integer $j$ we may let $D_{j}=\odot_{n}^{-1}(U)$ and we are through; and so, suppose that, for each $n$,

Received by the editors September 4, 1970.

AMS 1969 subject classifications. Primary 5420, 5425.

Key words and phrases. Product spaces, normality, countable paracompactness.

Copyright @ 1971, American Mathematical Society 
$\operatorname{cl} P_{n}(H) \cap \operatorname{cl} P_{n}(K) \neq \varnothing$. For each integer $n$, let $L_{n}=\operatorname{cl} P_{n}(H)$ $\cap \mathrm{cl} \mathcal{P}_{n}(K)$. Then $\left\{\mathcal{P}_{n}^{-1}\left(L_{n}\right)\right\}$ is a monotonic decreasing sequence of closed subsets of $X^{\omega}$ with no common part; and so, according to Lemma $\mathrm{A}$, there is a sequence $\left\{U_{n}\right\}$ of open subsets of $X^{\omega}$ such that (1) for each $n, \mathcal{P}_{n}^{-1}\left(L_{n}\right) \subset U_{n}$ and (2) $\bigcap_{i=1}^{\infty} \mathrm{cl} U_{i}=\varnothing$. For each $n$, let $V_{n}=\mathcal{P}_{n}\left(U_{n}\right)$. Then $\bigcap_{i=1}^{\infty} \mathrm{cl} \mathcal{P}_{n}^{-1}\left(V_{n}\right)=\varnothing$ and for each $n, V_{n}$ contains $L_{n}$. Now, for each integer $n,\left[\operatorname{cl} \rho_{n}(H)-V_{n}\right] \cap\left[\operatorname{cl} \rho_{n}(K)-V_{n}\right]=\varnothing$; and so, there is an open set $W_{n}$ in $X^{n}$ such that cl $P_{n}(H)-V_{n} \subset W_{n}$ $\subset \operatorname{cl} W_{n} \subset \operatorname{cl~} \odot_{n}(K)-V_{n}$. For each integer $n$, let $D_{n}=\odot_{n}^{-1}\left(W_{n} \cup V_{n}\right)$. Clearly, for each integer $n, H \subset D_{n}$. It remains to show that if $x$ is a point of $K$, then $x$ is not in $\bigcap_{i=1}^{\infty} \mathrm{cl} D_{i}$. To this end, suppose that $x \in\left[\cap_{i=1}^{\infty} \operatorname{cl} D_{i}\right] \cap K$. There is an integer $n$ so that $x$ is not in $\operatorname{cl}_{n}^{-1}\left(V_{n}\right)$. Thus, $P_{n}(x)$ is in $\mathrm{cl} P_{n}(K)-V_{n}$. It follows that $P_{n}(x)$ is not in cl $W_{n}$; and so, $x$ is not in $\mathrm{cl} D_{n}$ which is a contradiction from which it follows that $K \cap\left[\bigcap_{i=1}^{\infty} \operatorname{cl} D_{i}\right]=\varnothing$.

Now, suppose that $X^{\omega}$ is normal but $X^{\omega}$ is not countably paracompact. According to our Lemma A, there is a monotonic decreasing sequence $\left\{H_{n}\right\}$ of closed subsets of $X^{\omega}$ with no common part such that if $\left\{D_{n}\right\}$ is a sequence of open sets such that $D_{n}$ contains $H_{n}$ for each $n$, then $\operatorname{ncl} D_{n} \neq \varnothing$. Let $x$ and $y$ denote distinct parts of $X$ and let $x^{\omega}\left(y^{\omega}\right)$ denote that point of $X$ each coordinate of which is $x$ ( $y$, resp.). Let

$$
H=\bigcup_{n=1}^{\infty}\left[\left(\operatorname{cl} P_{n}\left(H_{n}\right)\right) \times x^{\omega}\right] \text { and } K=\bigcup_{n=1}^{\infty}\left[\left(\operatorname{cl} P_{n}\left(H_{n}\right)\right) \times y^{\omega}\right] .
$$

Then $H$ and $K$ are mutually exclusive closed subsets of $X^{\omega}$. Since $X^{\omega}$ is normal, there are open sets $U$ and $V$ in $X^{\omega}$ such that cl $U \cap \mathrm{cl} V=\varnothing$ and such that $H \subset U$ and $K \subset V$. Note that for each $n$, cl $\odot_{n}\left(H_{n}\right) \subset \odot_{n} U$ and $\mathrm{cl} \odot_{n}\left(H_{n}\right) \subset \odot_{n} V$. For each integer $n$, let $D_{n}$ $=\odot_{n}^{-1}\left(\odot_{n} U \cap \rho_{n} V\right)$. Now, $\bigcap_{n=1}^{\infty} \mathrm{cl} D_{n} \subset \operatorname{cl}(U \cap V)=\varnothing$ which is a contradiction from which the theorem follows. The following lemma was proved in [5]:

Lemma C. The space $X$ is perfectly normal if and only if for each closed set $H$ there is a sequence $\left\{D_{n}\right\}$ of open sets such that $H=\bigcap_{n=1}^{\infty} D_{n}$ $=\bigcap_{n=1}^{\infty} \operatorname{cl} D_{n}$.

Theorems B-D (and the methods of proof) should be compared to the results in $[3]$.

THEOREM B. If $X \times Y$ is hereditarily countably paracompact, then either $Y$ is perfectly normal or every countable discrete subspace of $X$ is closed in $X$.

Proof. Suppose that $X \times Y$ is hereditarily countably paracompact 
and $M=\left\{x_{1}, x_{2}, \cdots\right\}$ is a countable discrete subspace of $X$ with a limit point $x_{0}$. Let $H$ denote a closed subset of $Y$. Let $M_{1}$ $=\left\{(x, y) \subset X \times Y \mid x=x_{0}, y \in Y-H\right\}$ and $Z=(M \times Y) \cup M_{1}$. For each integer $n$, let $K_{n}=\left\{(x, y) \in Z \mid x=x_{n}, y \in H\right\}$ and $H_{n}=\bigcup_{i=n}^{\infty} K_{i}$. Then $\left\{H_{n}\right\}$ is a monotonic decreasing sequence of closed subsets of $Z$ with no common part. Since $Z$ is countably paracompact, by Lemma A, there is a sequence $\left\{U_{n}\right\}$ of open sets in $Z$ such that $\bigcap_{i=1}^{\infty} \mathrm{cl} U_{i}=\varnothing$ and $H_{n} \subset U_{n}$ for each $n$. For each integer $n$, let $D_{n}$ $=\left\{y \in Y \mid\left(x_{n}, y\right) \in \bigcap_{i=1}^{n} U_{i}\right\}$. Clearly, $H \subset D_{n}$ for each $n$. Suppose that $y \in(Y-H) \cap \bigcap_{i=1}^{\infty} \operatorname{cl} D_{i}$. Then $\left(x_{0}, y\right)$ is a point of $Z$ that is in $\bigcap_{i=1}^{\infty} \mathrm{cl} U_{i}$ which is a contradiction. The theorem now follows from Lemma $C$.

TheOREM C. The compact space $X$ is metrizable if and only if $X^{3}$ is hereditarily countably paracompact.

Proof. It need only be shown that if $X^{3}$ is hereditarily countably paracompact, then $X$ is metrizable. Since $X^{3}$ is homeomorphic to $X \times X^{2}$, it follows from Theorem B that $X^{2}$ is perfectly normal. Thus, $X$ is metrizable since it is compact and has a $G_{\delta}$-diagonal.

Theorem D. $X^{\omega}$ is perfectly normal if and only if $X^{\omega}$ is hereditarily countably paracompact.

Proof. Since any perfectly normal space is hereditarily countably paracompact [3], we need only show that if $X^{\omega}$ is hereditarily countably paracompact, then $X^{\omega}$ is perfectly normal. To this end, let $x$ and $y$ denote distinct points of $X$. For each integer $n$, let $Z_{n}$ $=x^{n} \times y^{\omega}$; i.e., each of the first $n$ coordinates is $x$ and each remaining coordinate is $y$. Then the set $\left\{Z_{1}, Z_{2}, \cdots\right\}$ is a discrete subspace of $X^{\omega}$ that is not closed in $X^{\omega}$. Now, for each $n, X^{\omega}$ is homeomorphic to $X^{\omega} \times X^{n}$; and so, by Theorem $\mathrm{B}$, for each $n, X^{n}$ is perfectly normal. Thus, by $[3$, Theorem 2$], X^{\omega}$ is perfectly normal.

\section{REFERENCES}

1. C. H. Dowker, On countably paracompact spaces, Canad. J. Math. 3 (1951), 219-224. MR 13, 264.

2. T. Ishikawa, On countably paracompact spaces, Proc. Japan Acad. 31 (1955), 686-687.

3. M. Katětov, Complete normality of Cartesian products, Fund. Math. 35 (1948), 271-274. MR 10, 315.

4. E. Michael, Paracompactness and the Lindelöf property in finite and countable cartesian products (to appear).

5. P. Zenor, On countable paracompactness and normality, Prace Mat. 13 (1969), 23-32. MR $40 \# 1975$.

Auburn University, Auburn, Alabama 36830 